\title{
Avaliação da prevalência e do grau de conhecimento do cirurgião-dentista em relação às emergências médicas
}

\author{
Assessment of prevalence and knowledge level of dental surgeons \\ regarding medical emergencies
}

\author{
Maria Kateryne Fiuza* \\ Samara Tessaro Balsan* \\ José Luiz Bernardon Pretto** \\ Rodrigo Alberto Cenci* \\ Ferdinando de Conto ${ }^{* *+}$
}

\section{Resumo}

$O$ atendimento clínico odontológico certamente não está restrito a repercussões apenas na cavidade bucal. Devido ao aumento gradativo do número de indivíduos acometidos sistemicamente, as chances de ocorrer eventos emergenciais durante a prática odontológica crescem substancialmente. Sendo assim, os cirurgiões-dentistas devem estar adequadamente preparados para lidar com todos os eventos que podem acontecer no seu ambiente de trabalho. Objetivo: avaliar a prevalência e o conhecimento das emergências médicas na prática odontológica dos cirurgiões-dentistas da cidade de Chapecó-SC. Sujeitos e método: a amostra foi composta por 63 cirurgiões-dentistas atuantes no município de Chapecó-SC. O instrumento de coleta de dados foi um questionário estruturado com 18 questões. Os dados foram analisados através dos programas Microsoft Excel 2010 e SPSS-20. Para análise e associação foi utilizado o teste qui-quadrado. A margem de erro considerada foi 5,0\%. Resultados: a maioria dos profissionais (54\%) possuía treinamento de suporte básico de vida; $60 \%$ julgavam-se capacitados a diagnosticar uma emergência médica; $57 \%$ já passaram por um quadro de emergência médica, prevalecendo situações de lipotimia (23\%). Em relação ao arsenal de equipamentos e medicamentos presentes no ambiente odontológico, 17\% deixaram essas questões em branco. Quanto aos casos clínicos, o diagnóstico de hipertensão apresentou uma margem alta de erro (62\%). Conclusão: apesar de os profissionais, em sua maioria, alegarem possuir treinamento de suporte básico de vida e julgarem-se capacitados para diagnosticar um quadro emergencial, é nítida a falta de equipamentos e medicamentos emergenciais nos consultórios odontológicos e foi preocupante o número de erros quanto ao diagnóstico dos casos clínicos.

Palavras-chave: Emergências. Prevalência. Síncope.

\section{Introdução}

A Odontologia, atualmente, é uma profissão que possui avanços significativos a respeito das diversas técnicas e materiais utilizados em seus procedimentos clínicos. Contudo, os avanços que a envolvem não excluem a possibilidade da ocorrência de situações inesperadas, mais precisamente situações de emergências médicas que podem transcorrer durante 0 atendimento ${ }^{1}$.

$\mathrm{O}$ atendimento clínico odontológico, certamente, não está restrito a repercussões apenas na cavidade bucal. Também envolve respostas reacionais do sis-

Acadêmica de Odontologia da Universidade Comunitária da Região de Chapecó (Unochapecó), Chapecó, SC, Brasil.

Mestre em Cirurgia e Traumatologia Bucomaxilofacial e professor da Faculdade de Odontologia da Unochapecó, Chapecó, SC, Brasil.

Mestre e doutor, Departamento de Cirurgia e Traumatologia Bucomaxilofacial do Hospital São Vicente de Paulo e professor da Faculdade de Odontologia da Universidade de Passo Fundo, Passo Fundo, RS, Brasil. 
tema geral do paciente. A maioria das pessoas em atendimento demonstra um determinado grau de ansiedade capaz de causar-lhes alterações sistêmicas, podendo ou não evoluir para uma emergência médica, que ocorre de maneira súbita e imprevisível ${ }^{2,3}$.

A emergência é caracterizada como sendo um evento que apresenta um risco imediato de morte ou, ainda, que poderá provocar lesões irreparáveis. $\mathrm{O}$ acontecimento desse evento sofre influência direta das causas primárias, como, por exemplo, a tensão emocional frente ao atendimento, e das secundárias, que englobam a faixa etária em que o paciente se insere ${ }^{4}$.

Obter o diagnóstico precoce das alterações sistêmicas que atingem o paciente é de grande importância para minimizar os riscos de ocorrência de uma emergência médica durante o atendimento odontológico. Uma anamnese adequada contribuirá positivamente na identificação de um ou mais fatores de risco que acometam o paciente. Adotar essas medidas simples de prevenção pode aumentar significativamente a segurança clínica durante a realização do procedimento ${ }^{5-8}$.

Nesse contexto, o cirurgião-dentista deve estar ciente de que poderá ter, no seu ambiente de trabalho, pacientes potencialmente inclinados a desenvolver situações de emergências médicas, que muitas vezes não estão diretamente relacionadas ao tratamento odontológico. Faz-se, então, necessária a preparação técnica para o enfrentamento de qualquer evento emergencial que possa vir a ocorrer antes, durante ou após o atendimento odontológico ${ }^{1}$. É indiscutível que o preparo dos profissionais deve iniciar durante o período da graduação e, ainda, que esse conhecimento deve ser reciclado em cursos de pós-graduação e aprimorado em cursos específicos de suporte básico de vida $(\mathrm{SBV})^{1}$.

A falta de estudos no âmbito de emergências médicas durante a prática odontológica leva a que seu esclarecimento ainda seja precário para muitos profissionais da área. Mesmo alguns estudos evidenciando que a maioria dessas emergências não representa perigo de morte para a vítima, é mandatório que os CDs estejam aptos a lidar com um evento emergencial, pois apenas esse preparo poderá contribuir para a diminuição da morbidade e mortalidade nessa situação $0^{9,10}$.

Em vista do exposto, este estudo pretende avaliar a prevalência e identificação das emergências médicas na prática odontológica dos CDs no município de Chapecó-SC.

\section{Sujeitos e método}

Este estudo foi realizado no município de Chapecó-SC no segundo semestre de 2012. A proposta foi enviada e aprovada pelo Comitê de Ética em
Pesquisa da Unochapecó no mês de agosto de 2012, número do registro: 168/12. Como instrumento de trabalho foi utilizado um questionário acompanhado de um termo de consentimento livre e esclarecido.

O presente estudo é do tipo retrospectivo, observacional descritivo, no qual se avaliou a prevalência das emergências médicas na prática odontológica e o conhecimento dos cirurgiões-dentistas diante destas. Foram selecionados cirurgiões-dentistas atuantes no município de Chapecó-SC. Para a seleção da amostra, foi solicitada ao Conselho Regional de Odontologia de Santa Catarina (CRO/SC) uma lista dos profissionais dentistas com seus respectivos endereços comerciais, totalizando 470 profissionais inscritos e ativos no referido conselho. Foram excluídos do estudo os profissionais que não atuam no município descrito.

Os questionários foram entregues via Google Docs e em cópias físicas aos 470 profissionais, obtendo-se uma amostra de 63 profissionais dentistas participantes do estudo. O questionário continha questões referentes ao assunto de emergências médicas no consultório odontológico, objetivando identificar sua prevalência e o grau de conhecimento dos CDs diante dessas intercorrências. O questionário, composto por dezessete perguntas "fechadas", contendo como resposta sim/não ou múltipla escolha, abordou variáveis como presença ou ausência de especialidade, se tinham ou não o treinamento de SBV e onde o adquiriram, preparo da equipe auxiliar para o manejo das emergências médicas, capacidade para diagnosticar um evento emergencial, frequência das emergências durante os atendimentos, presença de equipamentos e medicamentos de emergência nos consultórios odontológicos. Também foi analisado o grau de conhecimento dos profissionais a partir da elaboração de casos clínicos sobre o assunto abordado. Apenas uma questão era do tipo "aberta" e referente ao tipo de procedimento que estava sendo realizado durante a ocorrência do evento emergencial.

Os resultados foram compilados no programa Microsoft Excel 2010 e analisados no SPSS versão 20, 2012. Foram construídos tabelas e gráficos com frequência relativa e absoluta das respostas para cada questionamento. As respostas dos especialistas foram comparadas com as dos clínicos gerais com o teste estatístico qui-quadrado e foi considerado significativo $\mathrm{p}<0,05$.

\section{Resultados}

As características da amostra resultaram em $56 \%$ de cirurgiões-dentistas do sexo masculino, tendo $41 \%$ obtido o título entre os anos de 1993 e 2002. A maioria dos entrevistados tinha algum tipo de especialização $(65 \%)$. 
Quando questionados se possuíam treinamento de suporte básico de vida (SBV), 54\% ( $\mathrm{n}=34)$ dos profissionais responderam que sim e $46 \%(\mathrm{n}=29)$ relataram que não (Fig. 1).

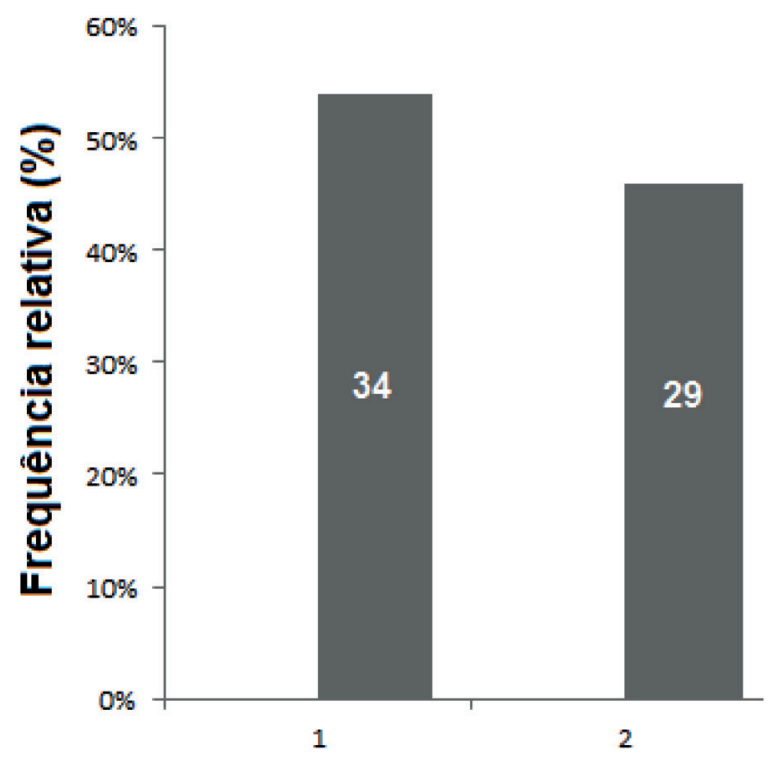

Figura 1 - Frequência relativa e absoluta do relato de treinamento prévio quanto a suporte básico de vida

Quinze entrevistados (19\%) relataram que o conhecimento foi adquirido durante a graduação e 13 (17\%), durante cursos extracurriculares (Fig. 2).

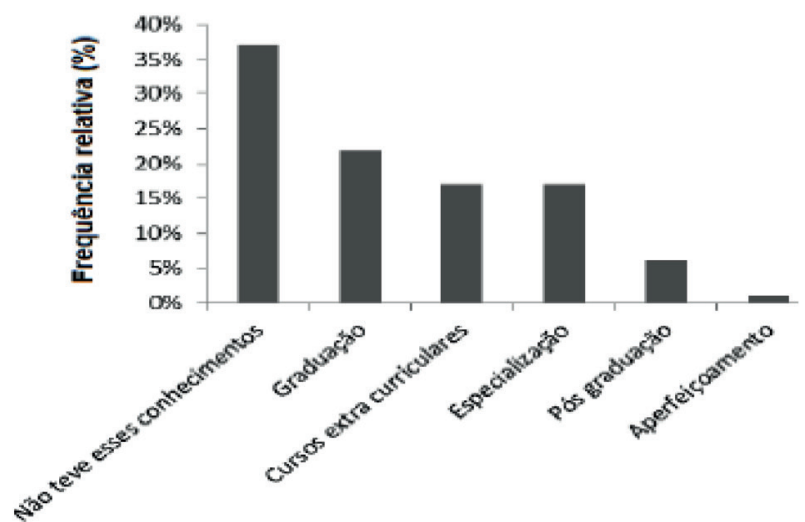

Figura 2 - Frequência de respostas para a pergunta: Onde adquiriu o conhecimento sobre suporte básico de vida (SBV)?

Em relação ao seu pessoal auxiliar, 84\% da amostra respondeu que os profissionais contratados em seus serviços não possuem treinamento de SBV, e apenas $14 \%$ da amostra afirmou que sua equipe odontológica o possui.

Quando questionados se julgavam-se capacitados para diagnosticar uma emergência médica no ambiente de trabalho, 60\% dos CDs responderam que sim (Fig. 3).

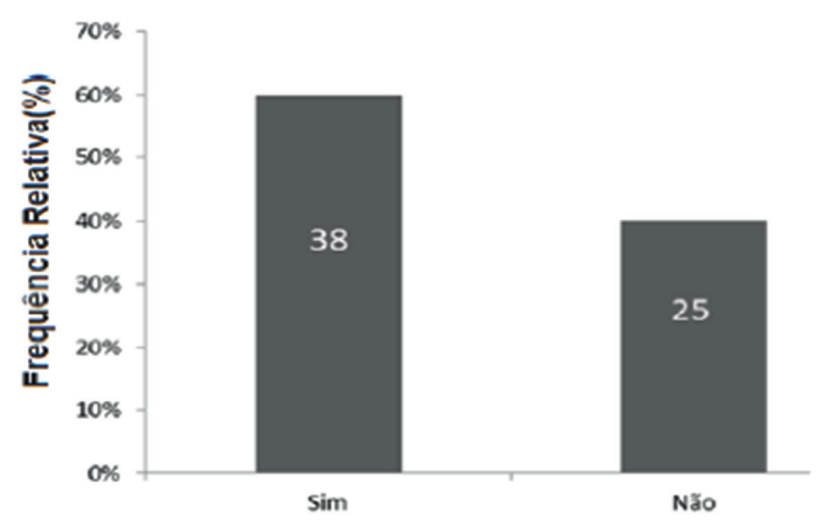

Figura 3 - Frequência de respostas para a pergunta: $C D$ se julga capacitado para diagnosticar uma emergência médica?

Os especialistas não se julgavam mais capacitados do que os clínicos gerais para diagnosticar um quadro emergencial ( $62 \%$ × 57\%, $p=0,716)$, assim como não apresentaram maior histórico de treinamento em SBV (45\% x 48\%, p = 0,858) (Tab. 1).

\begin{tabular}{|c|c|c|c|c|}
\hline Pergunta & Resposta & $\begin{array}{c}\text { Espacialista } \\
n=42\end{array}$ & $\begin{array}{c}\text { Clínico geral } \\
n=21\end{array}$ & $\mathrm{p}$ \\
\hline $\begin{array}{l}\text { Você se julga capacitado } \\
\text { para diagnosticar uma } \\
\text { emergência médica? }\end{array}$ & $\begin{array}{l}\text { Sim } \\
\text { Não }\end{array}$ & $\begin{array}{l}26(62 \%) \\
16(38 \%)\end{array}$ & $\begin{array}{c}12(57 \%) \\
9(43 \%)\end{array}$ & 0,716 \\
\hline Possui treinamento SBV? & $\begin{array}{l}\text { Sim } \\
\text { Não }\end{array}$ & $\begin{array}{l}23(55 \%) \\
19(45 \%)\end{array}$ & $\begin{array}{l}11(54 \%) \\
10(48 \%)\end{array}$ & 0,858 \\
\hline
\end{tabular}

A respeito da frequência das emergências médicas no consultório odontológico, $57 \%$ dos dentistas entrevistados já tiveram um evento emergencial durante 0 atendimento (Fig. 4).

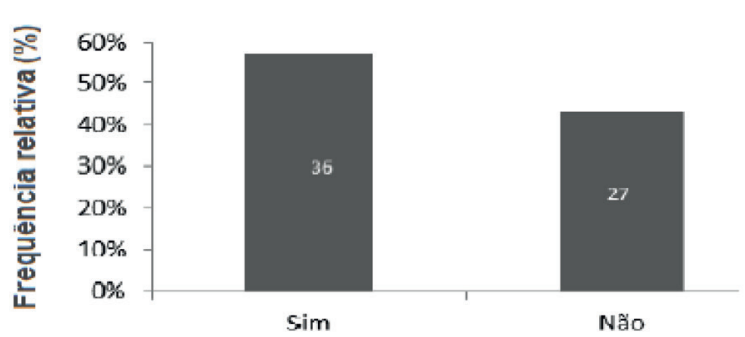

Figura 4 - Frequência de emergências durante os atendimentos

Das 104 emergências relatadas, a mais prevalente foi o quadro de lipotimia (23\%), seguida de medo da anestesia (14\%). Na Figura 5, podem ser observadas todas as emergências descritas nos questionários analisados. 


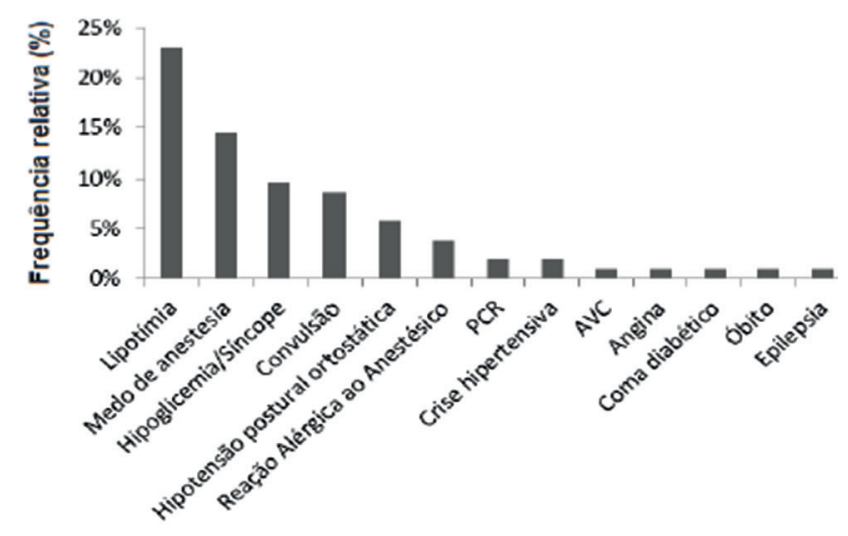

Figura 5 - Percentual dos tipos de emergência em relação ao total de emergências

Quando interrogados sobre o tipo de procedimento que estava sendo realizado durante a ocorrência do quadro emergencial, $25 \%$ da amostra referiu procedimento cirúrgico, totalizando a maioria das respostas.

Em relação ao arsenal de materiais/equipamentos/instrumentais para atendimento de emergência presentes nos consultórios odontológicos, $17 \%$ dos entrevistados não responderam à pergunta. Dos materiais citados como presentes nos consultórios, seringas e agulhas descartáveis e cânulas de traqueia e faríngea representam os mais e menos citados (63\% e 1\%), respectivamente. Na Figura 6, podem ser observados em cinza escuro o percentual de cada equipamento presente nos consultórios, em cinza claro (ao centro) o percentual de profissionais que não possuíam cada equipamento e em cinza médio (mais acima) os que deixaram a questão em branco.

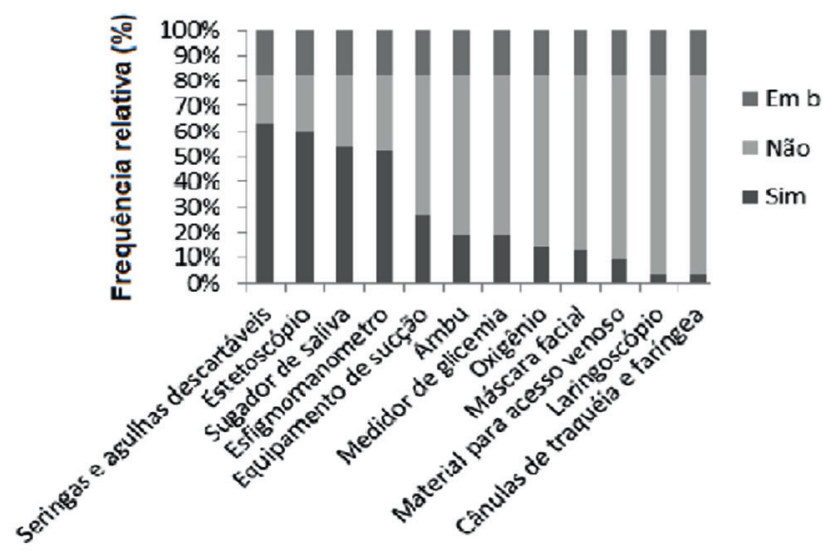

Figura 6 - Frequência relativa dos equipamentos disponíveis nos consultórios

Na pergunta sobre quais medicamentos estavam disponíveis nos serviços dos CDs, o medicamento mais citado foi Diazepam, em $48 \%$ dos casos, seguido de açúcares e afins (38\%). A frequência dos demais medicamentos pode ser observada na Figura 7 , registrando-se que $30 \%$ dos entrevistados deixaram essa questão em branco.

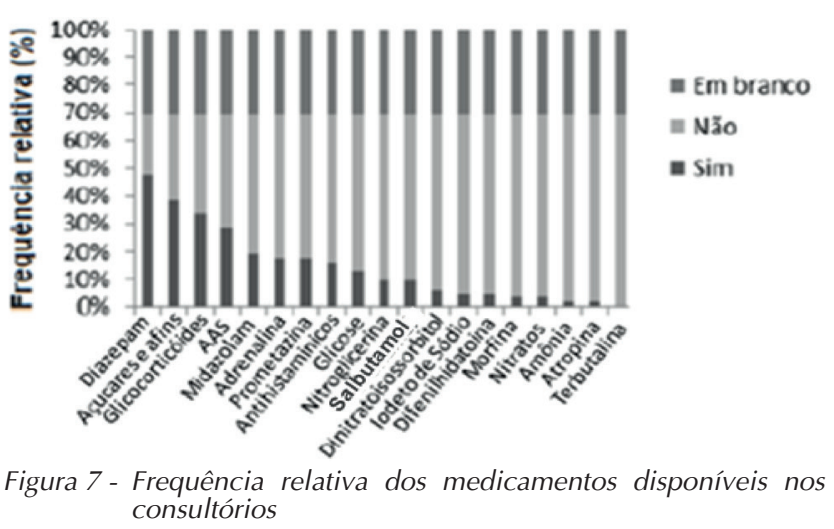

Em relação aos casos clínicos aplicados aos participantes do estudo, verificou-se que o diagnóstico de maior dificuldade foi o da hipertensão, que obteve uma frequência de $62 \%$ de erros, seguido do diagnóstico de convulsão, com $41 \%$ de respostas erradas. A pergunta relacionada a choque anafilático foi a que apresentou menor frequência de erro, com apenas $3 \%$.

\section{Discussão}

O cirurgião-dentista é um profissional da área da saúde que não está livre de ter seus pacientes acometidos por uma situação de emergência durante seus atos clínicos. A ocorrência dos eventos emergenciais durante a prática odontológica é rara, porém alguns fatores, como o aumento da expectativa de vida associado ao número crescente de portadores de doenças crônicas, podem facilitar o seu aparecimento.

A anamnese é um exame considerado indispensável em uma consulta odontológica, pois através dele os profissionais obtêm as principais informações necessárias para classificar o grau de risco do seu paciente. Informações relacionadas à saúde geral e bucal do indivíduo devem ser fornecidas durante a anamnese ${ }^{4}$.

Além de uma anamnese detalhada, é indispensável que os profissionais capacitem-se para os momentos de crise durante um atendimento, por meio de um treinamento que promova um conhecimento teórico e prático sobre suporte básico de vida. Em relação ao SBV, apesar de a maioria dos participantes deste estudo afirmar que apresentam a formação adequada para o manejo das emergências médicas, é preocupante que alguns profissionais ainda não possuam esse treinamento, por ser esse um requisito fundamental para garantir que o profissional consiga reconhecer uma situação emergencial e agir adequadamente durante sua ocorrência.

O diagnóstico preciso e correto das emergências médicas requer que o profissional possua um treinamento específico para tal. No entanto, verificou-se uma relação direta entre a percentagem dos profissionais que realizaram a formação comple- 
mentar e a capacidade dos profissionais de reconhecerem essas intercorrências.

Contrariamente aos resultados apresentados acima, Ricci et al. ${ }^{11}$ (1989) publicaram que $25 \%$ dos CDs consideravam-se capazes de diagnosticar um quadro emergencial. Na pesquisa de Santos e $\mathrm{Ru}$ mel $^{12}$ (2006), por sua vez, que 76,9\% das respostas informavam que os CDs não se sentiam aptos para o diagnóstico.

Em relação ao nível de formação dos profissionais dentistas, não se obteve uma diferença estatisticamente significante quando se comparou o nível de formação do profissional entre os clínicos gerais ou os especialistas e a sua capacidade para realizar o diagnóstico de uma emergência médica. Também não houve diferenças significativas entre os que possuíam e os que não possuíam o treinamento de SBV. Esse resultado, associado à falta de conhecimento por parte dos profissionais sobre o assunto, demonstra que estes estão saindo da graduação despreparados e que o conhecimento não está sendo adquirido de forma adequada durante o período de pós-graduação ${ }^{13}$. O tópico de emergências médicas deve ser abordado durante a graduação e reciclado durante a pós-graduação e cursos extracurriculares para que os profissionais consigam manter um nível de conhecimento adequado sobre 0 assunto rotineiramente ${ }^{14}$.

A maioria dos profissionais que participaram do estudo e que possuem conhecimento sobre suporte básico de vida alegou tê-lo adquirido durante a graduação. Porém, sabe-se que nesse nível de ensino ainda não existem conteúdos específicos, incluídos na matriz curricular do curso de Odontologia, que se disponham a treinar os acadêmicos em relação às emergências médicas ${ }^{15,16}$. Sendo assim, existe a necessidade de atualizações específicas desses conhecimentos após a graduação.

Em relação ao preparo da equipe auxiliar odontológica frente às emergências médicas, nosso estudo vai ao encontro das informações obtidas em Santos e Rumel ${ }^{13}$ (2006), em que 88,7\% das THDs ou ACDs também não possuíam o treinamento de SBV. Esses resultados representam mais uma deficiência encontrada na realidade da profissão, pois se sabe que um consultório odontológico com pessoal treinado e capacitado para os eventos emergenciais é capaz de realizar um planejamento adequado para agir durante um quadro emergencial, e, se a vida do paciente for perdida, toda a equipe odontológica tem direito a proteção legal, na medida em que realizaram de maneira correta todas as manobras necessárias para tentar reverter o caso ${ }^{17,18}$.

Apesar de incomum, a ocorrência de um evento emergencial durante 0 atendimento clínico foi relatado pela maioria dos profissionais, corroborando o estudo realizado por Veiga ${ }^{10}$ (2012), no qual a presença das emergências médicas durante a prática odontológica foi citada por $67 \%$ dos dentistas entrevistados.
Demonstra-se, assim, a necessidade de o profissional manter-se atualizado sobre esse importante tema, tanto de maneira técnica quanto adquirindo o conhecimento cientifico necessário para atuar nesse tipo de situação, evitando problemas de ordem ética e legal que possam aparecer devido a alguma conduta inadequada de sua parte ${ }^{13,19}$.

Dentre as emergências que podem estar presentes durante a prática odontológica, cita-se as situações de síncope/lipotimia, convulsão, reação alérgica, obstrução das vias aéreas, crises de asma, entre outras ${ }^{15}$, sendo o estresse, o medo ${ }^{15,20}$ e o uso de medicamentos específicos para algum comprometimento sistêmico que o paciente possui ${ }^{20}$ os principais agentes desencadeadores da maioria dos quadros emergenciais.

No presente estudo, a lipotimia foi o quadro emergencial mais prevalente. Outros autores obtiveram o mesmo resultado, no qual as situações de lipotimia e síncope também foram as mais citadas pelos profissionais entrevistados ${ }^{1,13,21,22}$. Mesmo não sendo comuns, situações de óbito podem estar acontecendo durante a prática odontológica, como foi demonstrado nos resultados do presente estudo. Portanto, é dever do profissional dentista estar adequadamente preparado para evitar que um quadro emergencial evolua a ponto de causar a morte do seu paciente.

Dentre os procedimentos odontológicos capazes de desencadear um quadro emergencial, pode-se destacar os procedimentos cirúrgicos como sendo os mais suscetíveis para iniciar tal evento. Esse resultado está de acordo com aqueles encontrados na literatura $^{21,23,24}$, os quais afirmam que os procedimentos cirúrgicos são os mais prevalentes para o aparecimento de um quadro emergencial. Isso se justifica pelo aumento do nível de estresse emocional enfrentado pelo paciente, uma vez que esses são procedimentos que exigem maior tempo clínico, e pela associação de medicamentos no período transoperatório.

Relacionado ao arsenal de medicamentos de emergência presentes nos consultórios, apenas alguns medicamentos foram "citados", e um número significativo de CDs (30\%) deixou essa questão em branco. Sabendo que medicamentos como adrenalina, atropina, nitratos, anti-histamínicos, corticoides, antieméticos, analgésicos potentes, entre outros, devem fazer parte do arsenal de medicamentos dos CDs, evidencia-se, com base nesses dados, que o despreparo por parte dos profissionais acontece também em relação aos medicamentos necessários para o manejo das emergências médicas.

No que concerne aos equipamentos emergenciais presentes, a negligência foi novamente evidenciada, pois a maioria dos profissionais não possui todos os equipamentos essenciais para o manejo de uma emergência médica. O consultório odontológico é considerado um ambiente cirúrgico, portanto deve 
ter, também, equipamentos e medicamentos específicos hospitalares, tais como AMBU (Sistema de Respiração Bolsa Máscara), laringoscópio, cânulas traqueais e agulhas para cricotireoideotomia ${ }^{25}$.

A análise do grau de conhecimento dos CDs quanto ao assunto de emergências médicas foi realizada pela inclusão de seis casos clínicos ao estudo. Os casos foram elaborados abordando algumas situações emergenciais que podem acontecer rotineiramente nos consultórios odontológicos, tais como crise hipertensiva, parada cardiorrespiratória, hipoglicemia, choque anafilático, convulsão e síncope. É digno de nota que o diagnóstico de maior dificuldade tenha sido o da hipertensão. Pelo fato de tal condição sistêmica estar presente em um número significativo de indivíduos que frequentam o ambiente odontológico, é preocupante que os profissionais dentistas não saibam reconhecer um caso de crise hipertensiva. Essa alteração sistêmica é simples e comum de ser diagnosticada quando o profissional possui um conhecimento adequado sobre os sinais e sintomas que predispõem a crise. Com base nisso, poderá ser estabelecida uma gestão preventiva da sua ocorrência, ao realizar a aferição regular da pressão sanguínea do paciente hipertenso antes de iniciar os procedimentos clínicos.

Para o presente estudo, não houve uma colaboração significativa por parte dos profissionais dentistas. Os questionários foram enviados aos 470 profissionais dentistas atuantes no município de Chapecó, obtendo-se apenas 63 questionários respondidos. Tal fato pode ser relacionado à falta de conscientização dos profissionais sobre o tema, bem como ao seu evidente despreparo para a atuação pronta e segura diante de emergências médicas.

Logo, tanto os acadêmicos em Odontologia quanto os profissionais precisam estar cientes que, para atuar na profissão, devem assumir responsabilidades que vão muito além de um tratamento odontológico. O CD deve tomar consciência de que, ao restringir sua atuação apenas para a cavidade oral, sem levar em conta o estado geral de saúde do seu paciente, poderá estar aumentando significativamente as chances de ocorrência de um evento emergencial. Esse fato, associado à falta de conhecimento adequado sobre o assunto, poderá gerar consequências negativas e até mesmo fatais para a vida do seu paciente.

\section{Conclusões}

Com base nos dados obtidos e pelo levantamento bibliográfico realizado, pode-se concluir que as emergências médicas estão cada vez mais frequentes no cotidiano do CD, devido, sobretudo, ao aumento gradativo de pacientes comprometidos sistemicamente que utilizam os serviços odontológicos. É perceptível a incapacitação dos CDs diante das emergências médicas, em razão, principalmente, da falta de formação para atuar nessas situações, bem como da negligência quanto ao arsenal necessário para a reversão das emergências. Diante de todos esses fatos, é oportuno que se alerte para a necessidade da conscientização efetiva dos profissionais com relação à capacitação para a atuação em situações de emergências no ambiente de trabalho.

\section{Abstract}

Clinical dental care is certainly not limited to repercussions in the oral cavity only. The growing number of systemically affected individuals substantially increases the likelihood of emergency events occurring during dental practice. Thus, dental surgeons should be adequately prepared to deal with all types of events that might occur in their dental office. Objective: to assess the prevalence and knowledge of medical emergencies occurring during dental practice of dental surgeons in the city of Chapecó, state of Santa Catarina, Brazil. Subjects and method: the sample consisted of 63 dental surgeons working in Chapecó, state of Santa Catarina, Brazil. Data were collected using an 18-item structured questionnaire, and analyzed with the use of Excel 2010 and SPSS-20. The chi-square test was used for analyses and associations. The margin of error was 5.0\%. Results: most professionals had basic life support training (54\%), felt they were qualified to diagnose a medical emergency (60\%), and had already been faced with situations involving a medical emergency (57\%), especially syncopal episodes (23\%). Regarding the arsenal of devices and medications available in the dental environment, $17 \%$ of respondents left these questions blank. As for clinical cases, the diagnosis of hypertension had a high margin of error (62\%). Conclusions: although most dental surgeons claimed that they had basic life support training and were able to diagnose an emergency event, it is clear that there was a lack of equipment and drugs required for emergency situations in dental offices. Also worrying was the number of errors in the diagnosis of clinical cases.

Keywords: Emergencies. Prevalence. Syncope.

\section{Referências}

1. Caputo IGC. Emergências médicas em consultório odontológico: implicações éticas e legais para o cirurgião-dentista [Dissertação de Mestrado]. Piracicaba: UNICAMP/FOP; 2009.

2. Malamed SF. Emergency medicine: beyond the basic. J Amer Dent Assoc 1997; 128:843-54.

3. Caputo IGC, Bazzo GJ, Silva RHA, Daruge Júnior E. Vidas em risco: emergências médicas em consultório odontológico. Rev Cir Traumatol Buco-Maxilo-Fac 2010, 10(3):51-8.

4. Andrade ED, Ranali J. Emergências médicas em Odontologia. 2. ed. São Paulo: Artes Médicas; 2004.

5. Gaetti-Jardim EC, Pereira FP, Fattah CMR de S, Aranega AM. Prevalência e perfil epidemiológico das alterações sistêmicas em pacientes atendidos pelo serviço de cirurgia e traumatologia buco-maxilo-facial da Faculdade de Odon- 
tologia do Campus de Araçatuba - UNESP. Rev Odontol UNESP 2008; 37:191-6.

6. Colet D, Griza GL, Fleig CN, Cenci RA, Sinegalia AC. Acadêmicos e profissionais da odontologia estão preparados para salvar vidas? Rev Fac Odontol Unuv Passo Fundo 2011;16(1):25-9.

7. Romriell GE, Streeper SN. The medical history. Dent Clin North Am 1982; 26:3-11.

8. Sonis ST, Fazio RC, Fang L. Hipertensão. In: Sonis ST, Fazio RC, Fang L. Princípios e prática de medicina oral. 2. ed. Rio de Janeiro: Guanabara Koogan; 1996. p. 37-45.

9. Hupp JR. Avaliação do estado de saúde pré-operatório. In: Peterson LJ, Ellis III E, Hupp JR, Tucker MR. Cirurgia oral e maxilofacial contemporânea. 3. ed. Rio de Janeiro: Guanabara Koogan; 2000.

10. Veiga D, Oliveira R, Carvalho J, Mourão J. Emergências médicas em medicina dentária: prevalência e experiência dos médicos dentistas. Rev Port Estomatol Cir Maxilofac 2012; 53(2):77-82

11. Ricci A, Bijella VT, Moraes N. O cirurgião-dentista face às emergências médicas. Parte I. Avaliação da capacidade profissional. Rev Paulista Odontol 1989; 3(11):26-35.

12. Santos JC, Rumel D. Emergência médica na prática odontológica no estado de Santa Catarina: ocorrência, equipamentos e drogas, conhecimento e treinamento dos cirurgiões-dentistas. Ciênc Saúde Coletiva 2006; 11:183-90.

13. Nunes RJAA. Importância das emergências médicas para o cirurgião-dentista [Dissertação de Mestrado]. Paraíba: UFPB; 2010.

14. Arsati F, Montalli VA, Flório FM, Ramacciato JC, Da Cunha FL, Cecanho R, et al. Brazilian dentists' attitudes about medical emergencies during dental treatment. J Dent Educ 2010; 74:661-6.

15. Silva EL, Nogueira AJS. Suporte básico de vida: diferencial no atendimento ou responsabilidade legal odontológica? Revista APCD 2006; 60(5):392-4.

16. Carvalho C. Emergências médicas no atendimento odontológico. Rev Bras Odontol 2003; 60(2):108-11.

17. Peskin RM, Siegelman LI. Emergency cardiac care - oral, legal and ethical considerations. Dent Clin North Am 1995; 39:677-88.

18. Haas DA. Preparing dental office staff members for emergencies: developing a basic action plan. JADA 2010; 141(5 suppl):8S-13S

19. Merly F. O cirurgião-dentista e as emergências médicas no consultório: será que estamos preparados para enfrentar este problema? Rev Bras de Odontol 2010; 67(1):6-7.

20. Gomez RS, Maia DMF, Lehman LFC, Santoro DR, Azeredo P, Castro WH. Emergências médicas no consultório odontológico. Rev CROMG 1999; 5(1):4-10.

21. Malamed SF. Managing medical emergencies. J Am Dent Assoc 1993; 124(8):40-53.

22. Oliveira TC, Torriani MA, Figueiredo PJ. Avaliação do preparo dos cirurgiões-dentistas para o atendimento de urgências e emergências médicas no consultório. J Bras Clin Odontol Int Saúde Bucal Col 2006; 10(52):59-65.

23. Hupp JR. Prevenção e tratamento das emergências médicas. In: Ellis E, Hupp JR, Peterson LJ, Tucker MR. Cirurgia oral e maxilofacial contemporânea. 4. ed. Rio de Janeiro: Elsevier; 2005. p. 3-43.
24. Atherton GJ, Pemberton MN, Thornhill MH. Medical emergencies in general dental practice in reat Britain. Part I: their prevalence over a 10-year period. Br Dent J 1999; 186:72-9.

25. Marques IHS, Pacheco W. Emergência no consultório: quem está preparado? Rev ABO Nac 1999; 7(1):7-11.

Endereço para correspondência:

José Luiz Bernardon Pretto

Unochapecó - Universidade Comunitária da

Região de Chapecó

Rua Senador Atílio Fontana, 591-E

Bairro Efapi

89809-000 Chapecó, SC

Fone/Fax: (16) 3602 3969, 36024102

E-mail: jl.pretto@hotmail.com

Recebido: 05/08/2013. Aceito: 13/01/2014. 